\title{
APPROXIMATION OF $Z_{2}$-COCYCLES AND SHIFT DYNAMICAL SYSTEMS
}

\author{
I. Filipowicz, J. KWIATKowSKI, M. LEMAṄCZYK
}

\begin{abstract}
Let $\bar{G}=G\left\{n_{t}, n_{t} \mid n_{t+1}, t \geq 0\right\}$ be a subgroup of all roots of unity generated by $\exp \left(2 \pi i / n_{t}\right), t \geq 0$, and let $\tau:(X, B, \mu) \circlearrowright$ be an ergodic transformation with pure point spectrum $\bar{G}$. Given a cocycle $\varphi, \varphi: X \longrightarrow$ $\mathbf{Z}_{2}$, admitting an approximation with speed $0\left(1 / n^{1+c}, \epsilon>0\right)$ there exists a Morse cocycle $\psi$ such that the corresponding transformations $\tau_{\varphi}$ and $\tau_{\downarrow}$ are relatively isomorphic. An effective way of a construction of the Morse cocycle $\psi$ is given. There is a cocycle $\varphi$ oddly approximated with an arbitrarily high speed and without roots.

This note delivers examples of $\varphi$ 's admitting an arbitrarily high speed of approximation and such that the power multiplicity function of $t_{\infty}$ is equal to one and the power rank function is oscillatory. Finally, we also prove that if $\varphi$ is a Morse cocycle then each proper factor of $\tau_{\varphi}$ is rigid. In particular continuous substitutions on two symbols cannot be factors of Morse dynamical systems.
\end{abstract}

\section{Introduction and statement of results}

Let $T:(X, B, \mu) \circlearrowleft$ be an ergodic transformation of a Lebesgue space. We will denote by $S p(T)$ the group of all the eigenvalues of the unitary operator $U_{T}: L^{2}(X, \mu) \circlearrowleft, U_{T} f=f \circ T . T$ is said to have rational pure point spectrum (r.p.p.s) if $L^{2}(X, \mu)$ is generated by the eigenfunctions of $U_{T}$ and, besides,

$$
S p(T)=G\left\{n_{t}: t \geq 0\right\}
$$

where $G\left\{n_{t}: t \geq 0\right\}, \quad n_{t} \mid n_{t+1}, \quad t \geq 0$, denotes the subgroup of all roots of unity generated by $\exp \left(2 \pi i / n_{t}\right), \quad t \geq 0$.

Let $D^{n_{t}}=\left(D_{0}^{n_{t}}, D_{1}^{n_{t}}, \ldots, D_{n_{t}}^{n_{t}}\right)$ be a partition, i.e. $D_{i}^{n_{t}} \cap D_{j}^{n_{t}}=\emptyset, i \neq$ $j, D_{i}^{n_{t}} \in B$. Then $D^{n_{t}}$ is called a partition of $X$ if, besides, ${ }_{i=0}^{n_{t}} D_{i}^{n_{t}}=X$. We call $D^{n_{t}}$ a $T$-tower (with height $n_{t}$ ) if $T^{i} D_{o}^{n_{t}}=D_{i}^{n_{t}}, \quad i=0, \ldots, n_{t}-1$ and a $T$-tower of $X$ if, besides, $D^{n t}$ is a partition of $X$. The ergodicity of $T$ says that if $D^{n_{t}}$ is a $T$-tower of $X$ with height $n_{t}$ then this is the only $T$-tower of $X$ with that height (reordering elements of $D^{n_{t}}$, if necessary). It turns out (cf. [15]) that: 
(1) $T$ has r.p.p.s. with $S_{p}(T)=G\left\{n_{t}: t \geq 0\right\}$ iff there is a sequence $\left(D^{n_{t}}\right)_{t \geq 0}$ of $T$-towers of $X$ such that $D^{n}: \nearrow B$.

Let $K$ denote the class of all the $\mathbf{Z}_{2}$-cocycles of $(X, B, \mu)$ i.e. $\varphi \in \mathcal{K}$ if $\varphi: X \longrightarrow Z_{2}$ is measurable. We endow $K$ with the natural topology given by the metric

$$
\zeta\left(\varphi, \varphi^{\prime}\right)=\mu\left(\varphi^{-1}(0) \Delta \varphi^{\prime-1}(0)\right)+\mu\left(\varphi^{-1}(1) \Delta \varphi^{\prime-1}(1)\right) .
$$

With this metric $K$ becomes a complete, separable metric space. Having fixed $T:(X, B, \mu) \bigcirc$ we define the class $K_{T}$ of all $\mathbf{Z}_{2}$-extensions $T_{\varphi}, \varphi \in \mathcal{K}$, of $T$, i.e.

$$
T_{\varphi}:\left(X \times \mathbf{Z}_{2}, \tilde{B}, \tilde{\mu}\right) \circlearrowright, \quad T_{\varphi}(x, i)=(T x, \varphi(x)+i),
$$

where $\tilde{\mu}$ is the product measure $\mu \times \nu_{2} \quad\left(\nu_{2}(0)=\nu_{2}(1)=1 / 2\right)$ and $\tilde{B}$ is the corresponding product $\sigma$-algebra. A cocycle $\varphi$ is called ergodic whenever $T_{\varphi}$ is ergodic. Now, changing $T$ we obtain the class of all ergodic $\mathbf{Z}_{2}$-extensions of automorphisms with r.p.p.s.

This class is one of more interesting classes in ergodic theory. A great deal of the attention has been devoted to the study of it (for instance [1], [2], [6], $[10],[13],[14],[16],[18])$.

Assume $\left(D^{n_{+}}\right)$is a sequence of $T$-towers of $X$ arising from (1) and let $f: N \longrightarrow R$ be a real function. Following [7] we call $\varphi \in \mathcal{X}$ oddly (evenly) approximated with speed $o(f(n))$ if for some subsequence $\left\{n_{t_{k}}\right\}$ there exists sets $F_{k}$ consisting of an odd (even) number of members of $D^{n_{t_{k}}}$ such that

$$
\mu\left(\varphi^{-1}(1) \triangle F_{k}\right)=o\left(f\left(n_{t_{k}}\right)\right)
$$

The odd approximation with speed $o(1 / n)$ guarantees the ergodicity of $\varphi([\boldsymbol{7}])$.

Assume $\varphi \in K$ and $\left(D^{n_{t}}\right)$ is a sequence of $T$-towers given by (1). Then $\varphi$ is said to be a Morse cocycle if there is a subsequence $\left(n_{t_{k}}\right)$ such that $\varphi \mid D_{i}^{n_{t_{k}}}$ is constant $\left(\varphi \mid D_{i}^{n_{c_{k}}}=a_{i}^{t}\right)$ on each level of $D^{n_{i_{k}}}$ except for $i=n_{t_{k}}-1$. The main result of $[\mathbf{1 4}]$ was

Representation Theorem. If $\varphi$ is oddly approximated with speed $0\left\{\frac{1}{n^{2}}\right)$ then there is a Morse sequence $x=b^{0} \times b^{1} \times \ldots$ such that $T_{\varphi}$ is isomorphic to the Morse dynamical system determined by $x$. (We refer to $[8],[10],[12 /$ for the definition and properties of Morse sequences).

In the present paper we stenghten the Representation Theorem proving:

Theorem 1. If $\varphi \in \mathcal{X}$ is ergodic and admits an odd (or even) approximation with speed $0\left(\frac{1}{n^{1+c}}\right), \epsilon>0$, then there exists a Morse cocycle $\psi$ such that $T_{\varphi}$ and $T_{\psi}$ are relatively isomorphic. 
We recall that $T_{\varphi}$ and $T_{\psi}$ are relatively isomorphic if there exists a cocycle $f: X \rightarrow Z_{2}$ such that

$$
\varphi(x)+f(T x)=f(x)+\psi(x)
$$

Then the map $I(x, i)=(x, f(x)+i)$ establishes an isomorphism between $T_{\varphi}$ and $T_{\psi}$.

We have been unable to decide whether or not the Theorem holds for $\epsilon=0$.

Looking at the proof of the Representation Theorem we see that it does not provide any effective way of a construction of the Morse cocycle $\psi$ (or the Morse sequence $x$ ). The proof of Theorem 1 is based on a quite different idea and allows to determine the Morse cocycle in an algorithmic way.

Let $G$ be the group of all $n_{t}$-adic integers i.e.

$$
G=\left\{g ; g=\sum_{0}^{\infty} g_{t} \cdot n_{t-1}, 0 \leq g_{t} \leq \lambda_{t}-1, n_{-1}=1\right\}, \lambda_{t}=\frac{n_{t+1}}{n_{t}}, t \geq 0,
$$

and let $T$ be the translation on the unit element $\hat{1}$. Then $(G, T, m),(m$ is the Haar measure) is an ergodic system having r.p.p.s., $S_{p}(T)=G\left\{n_{t}, t \geq 0\right\}$. Hence if $\varphi: G \rightarrow Z_{2}$ is a cocycle satisfying the assumptions of Theorem 1 then it can be modyfied by a coboundary cocycle getting $\psi$, which is measurable with respect to the algebra generated by $\left\{D^{n} t\right\}, t \geq 0$. Although $\psi$ cannot be continuous on $G$ (except for some trivial cases), there is a metod making such cocycles continuous. Namely, $\psi$ is the so called Toeplitz cocycle in the sense of [13] i.e. it is completely determined by some Toeplitz sequence $\eta \in\{0,1\}^{Z}$. If we take $X=\overline{\theta(\eta)}$ (the closure of the trajectory of $\eta$ via the shift $r$ ) and the cocycle $\psi^{\prime}: \overline{\theta(\eta)} \longrightarrow \mathbf{Z}_{2}, \psi^{\prime}(y)=y[0]$, then the automorphisms $\tau_{\psi} \psi^{\prime}$ and $T_{\psi}$ are metrically isomorphic $([13])$. In other words there is some effective way of a construction of an ergodic $r_{\psi}$ ' (with $\psi^{\prime}$ to be continuous) which is isomorphic to $T_{\psi}$.

Notice that from $[4]$ it follows that there is a topological process $\{\tilde{G}, \tilde{T}, \tilde{\mu}\rangle$, a metric isomorphism $\Pi:(\tilde{G}, \tilde{\mu}) \rightarrow(G, \mu) \Pi \tilde{T}=T \Pi$ and a continuous function $\tilde{\varphi}: \tilde{G} \rightarrow \mathbf{Z}_{2}$ such that $\tilde{\varphi}=\varphi \circ \Pi$ a.e. This implies that $T_{\varphi}$ and $\widetilde{T}_{\bar{\varphi}}$ are isomorphic. In particular, if $\varphi$ is a Toeplitz cocycle then $\tilde{G}=\overline{\theta(\eta)}, \tilde{T}=r$. The task arises how to determine $(\tilde{G}, \tilde{T})$ (in an effective way) for a general $\varphi \in K$. In particular it would be interesting to know whether given $\varphi: G \rightarrow \mathbf{Z}_{2}$ there exists a Toeplitz cocycle $\psi$ such that $r_{\psi}$ is isomorphic to $T_{\varphi}$. If this is the case we would have $G=\overline{\theta(\eta)}$. Our paper delivers a construction of such a $\psi$ if $\varphi$ fullifils the assumptions of Theorem 1.

In the remainder of the paper we consider some problems concerning cocycles admitting a high speed of approximation. For instance, it turns out that for any $T$ 
(4) there is a cocycle $\varphi$ oddly approximated with an arbitrarily high speed and without roots.

The next application is connected with the oscillation of the rank power function. Let $U:(Y, \tau, \nu) O$ be an ergodic automorphism. For the definition of the rank ( $\mathrm{rk}(U))$ we refer to $[3],[9]$ and to [19] for the definition of the maximal spectral multiplicity (m.s.m.(U)). These notions allow to define two functions

$$
s \longrightarrow \operatorname{rk}\left(U^{s}\right), \quad s \longrightarrow \operatorname{m.s.m.}\left(U^{s}\right), \quad s=1,2, \ldots
$$

called power rank function and power multiplicity function respectively. They are defined for those s's that $U^{s}$ is ergodic. In [9] J. King raised the question whether the power rank function had to be monotonic (it is rather easy to see that $\operatorname{rk}(U) \leq \operatorname{rk}\left(U^{\circ}\right), s \geq 1$ ). Then in [3] there is an example for which the power rank function is oscillatory. However, $\mathrm{rk}(U) \geq \mathrm{m} . \mathrm{s.m} .(U)$ and this example is based on the following facts: for some subsequences $\left(n_{k}\right),\left(m_{k}\right) \operatorname{rk}\left(U^{n_{k}}\right)=$ 1 and m.s.m. $\left(U^{m_{k}}\right) \geq 2$. This note delivers examples of $\psi^{\prime}$ 's addmitting an arbitrarily high speed of approximation with

$$
\begin{gathered}
\operatorname{sk}\left(\left(T_{\varphi}\right)^{s}\right)= \begin{cases}1, & \text { g.c.d. }\left(s, n_{t}\right)=1, s-\text { odd } \\
2, & \text { g.c.d. }\left(s, n_{t}\right)=1, s-\text { even }\end{cases} \\
\text { m.s.m. }\left(\left(T_{\varphi}\right)^{s}\right)=1, \quad \text { g.c.d. }\left(s, n_{t}\right)=1 .
\end{gathered}
$$

In [14] the author raised the factors problem for the class of Morse sequences, i.e. given a Morse cocycle $\varphi$ we seek all $T_{\varphi}$-invariant sub- $\sigma$-algebras $C \subset B$. Of course $B=\left\{A \times \mathbf{Z}_{2}, A \in B\right\}$ is an example of such a $C$. The action of $T_{\varphi}$ on $B$ is isomorphic to $T$. On the other hand the class of ergodic $\mathbf{Z}_{2}$-extensions of r.p.p.s. automorphisms is closed under taking factors. Is there a $C \subsetneq \tilde{B}$ such that action of $T_{\varphi}$ on $C$ (i.e. $\left.T_{\varphi}:\left(X \times \mathbf{Z}_{2}, C, \tilde{\mu}\right) 0\right)$ has partly continuous spectrum? We remark the following consequence of coding arguments used in [14] (compare it with the analogous result of J. King [9] for rank I class).

Theorem 2. If $\varphi$ is a Morse cocycle then each proper factor of $T_{\varphi}$ is rigid. In particular continuous substitutions [2/ on two symbols cannot be factors of Morse dynamical systems.

The natural reverse problem is the following. Can any rigid Morse dynamical system be extended to a Morse dynamical system with larger group of eigenvalues? There is a positive answer in case of sufficiently high speed of approximation (odd or even), but we have been unable to solve this problem in general. Has a positive answer analogous question for rank 1 class?

\section{Proof of Theorem 1}

Assume $T:(X, B, \mu) \bigcirc$ with r.p.p.s., $S_{p}(T)=G\left\{n_{t}: t \geq 0\right\}$ and let $\varphi \in \mathcal{K}$ admit an odd approximation with speed $0\left(\frac{1}{n^{1+c}}\right), \epsilon>0$. Hence there is a 
subsequence $\left\{n_{t_{k}}\right\}$ satisfying (2). For a simplication of notations we assume that this subsequence is equal to the $\left\{n_{t}\right\}$, i.e.

$$
n_{t}^{1+\varepsilon} \mu\left(\varphi^{-1}(1) \Delta F_{t}\right) \underset{t \rightarrow \infty}{\longrightarrow} 0
$$

where $F_{t}$ is a union of an odd number of levels of $D^{n_{t}}$. Assume that $\psi \in X$ is another ergodic cocycle. Then it is known that any isomorphism between $T_{\varphi}$ and $T_{\psi}$ is of the form $S_{\psi^{\prime}}:\left(X \times \mathbf{Z}_{z}, \tilde{B}, \tilde{\mu}\right) \circ$, where $S T=T S$ and

$$
\psi^{\prime}(T x)+\psi^{\prime}(x)=\varphi(x)+\psi(S x) \quad \text { in } ; \mathbf{Z}_{2}
$$

where $\psi^{\prime} \in K$ (see [17]). Let us notice that given $S,(7)$ has measurable solution iff $T_{\psi \circ \mathcal{S}+\varphi}$ is not ergodic. Indeed, $T_{\psi \circ S+\varphi}$ is ergodic iff there is no measurable solution $\xi: X \longrightarrow S^{1}$ such that

$$
\xi(T x)=(-1)^{(\psi \circ S+\varphi)(x)} \cdot \xi(x)
$$

(see $[\mathbf{7}],[\mathbf{1 8}])$. If $T_{\psi \circ S+\varphi}$ is not ergodic then we get a measurable solution of (8). Thus, the function $\xi(x)=\xi^{2}(x)$ is $T$-invariant, so by the ergodicity of $T$ it is constant. Therefore without loss of generality we can assume that $\xi(x)= \pm 1$. Then the function $\psi^{t}=1_{A}, A=\xi^{-1}(-1)$ is a solution of (7). On the other hand if $\psi^{\prime}$ is a solution of $(7)$ then the function $\xi(x)=(-1)^{\psi^{\prime}(x)}$ satisfies (8).

The following simple lemma will be useful in the proof of Theorem 1.

Lemma 1. Let $U:(Y, C, \nu) \circlearrowright$ be an automorphism of a Lebesgue space. Assume that there is a sequence $\left\{A_{n}\right\}, A_{n} \in C$ such that

$$
\nu\left(A_{n+1} \triangle A_{n}\right)<\epsilon_{n}, \quad \sum_{1}^{\infty} \epsilon_{n}<\infty
$$

$$
\nu\left(U A_{n} \Delta A_{n}\right) \underset{n}{\rightarrow} 0
$$

Then $U$ is not ergodic. 
Now, we turn back to the cocycle $\varphi$. Fix $t \geq 0$ and consider the tower $D^{n}$.

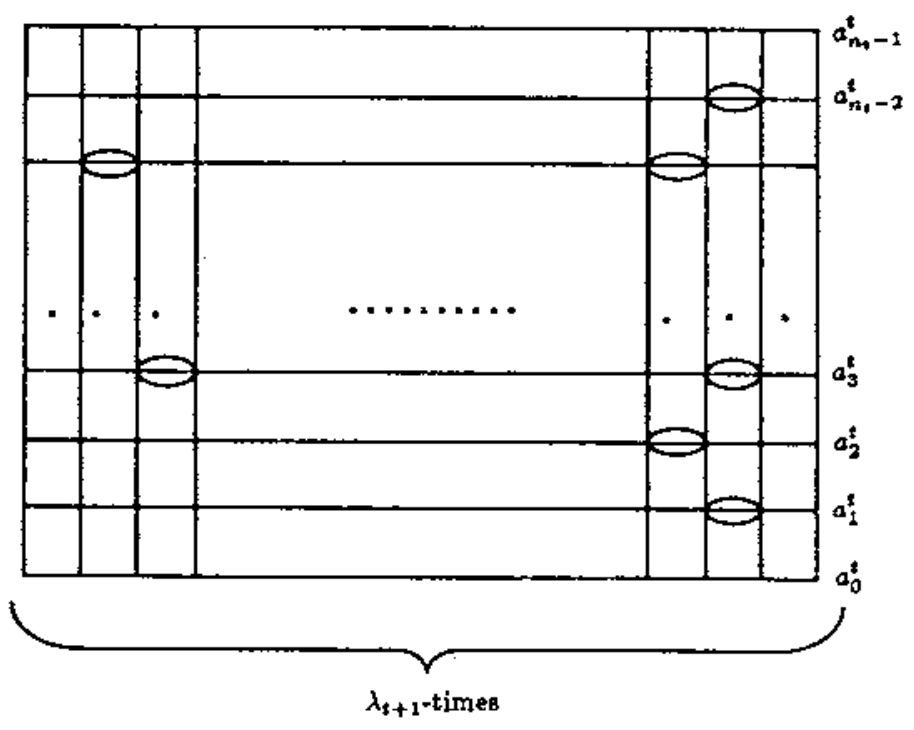

Picture I

Then the speed of approximation (which means that (6) holds) says that the function $\varphi$ restricted to each level $D_{i}^{n_{t}}, i=0,1, \ldots, n_{t}-1$, is "almost" constant, i.e.

$$
\left.\varphi\right|_{D_{i}^{m_{e}}=a_{i}^{t}}
$$

except for a part of $D^{n_{t}}$ with measure $\leq \frac{\epsilon_{t}}{n_{t}^{2+c}}, \quad \epsilon_{t} \rightarrow 0$. Because of the odd approximation,

$$
\sum_{i=0}^{n_{1}-1} a_{i}^{t}=1
$$

To construct $D^{n_{t+1}}$ we divide the tower $D^{n_{t}}$ into $\lambda_{t+1}$ columns with the same measure $1 / \lambda_{t+1}$ (Picture 1), $\lambda_{t+1}=n_{t+1} / n_{t}$. The $n_{t}$ pieces of the $s$-th column $\left(s=0,1, \ldots, \lambda_{t+1}-1\right)$ are the levels of $D^{n_{t+1}}$ assigned to the numbers $s \cdot n_{t}, s$. $n_{t}+1, \ldots, s \cdot n_{t}+n_{t}-1$.

We say that there is an error in $D_{j}^{n_{t+1}}, j=i+s \cdot n_{t}, 0 \leq i \leq n_{t}-1,0 \leq$ $s \leq \lambda_{t+1}-1$ if

$$
a_{i}^{t} \neq a_{j}^{t+1} \text {. }
$$

In Picture 1 such levels are marked by the sign " 0 ". Denote by $m_{t}$ the number of all columns with some errors. The measure $\mu$ of such a column is 
equal to $1 / \lambda_{t+1}$. Therefore the measure of all columns with errors is equal to

$$
C E^{t+1}=\frac{m_{t}}{\lambda_{t+1}} .
$$

Moreover, the measure $\mu$ of any error is equal to $1 / n_{t+1}$, so in view of (6)

$$
n_{t}^{1+\varepsilon} \cdot \frac{k_{t}}{n_{t+1}} \longrightarrow 0
$$

where $k_{t}$ is the number of all errors. But $m_{t} \leq k_{t}$ and the combination of (13) and (14) implies

$$
n_{t}^{\varepsilon} \cdot C E^{t+1} \underset{t}{\longrightarrow} 0
$$

and consequently

$$
\sum_{t=0}^{\infty} C E^{i+1}<\infty
$$

since $\varepsilon>0$.

Construction of a Morse cocycle. At each stage $t$ our cocycle $\psi$ will be constant on each level $D_{i}^{n_{t}}, i=0,1, \ldots, n_{t}-2$, and will not be defined on $D_{n_{t}-1}^{n_{t}}$. We define $\psi$ on $D^{n_{0}}$ in an arbitrary way and assume that $\psi$ is given on levels of $D^{n_{t}}, t \geq 0$, i.e.

$$
\left.\psi\right|_{D_{i}^{n_{t}}}=b_{i}^{t}, \quad i=0,1, \ldots, n_{t}-2 .
$$

First of all we define $b_{n_{t}-1}^{t}$ so that

$$
\sum_{i=0}^{n_{i}-1} b_{i}^{t}=1 .
$$

We do not change the function $\psi$ on the levels $D_{j \cdot n_{+}+i}^{n_{i}}, j=0,1, \ldots, \lambda_{t+1}-1$, $i=0, \ldots, n_{t}-2$, i.e. we put

$$
\psi \mid D_{j \cdot n_{t}+i}^{n_{t+1}}=b_{i}^{t} .
$$

Next we should define the function $\psi$ on the levels $D_{j \cdot n_{t}+n_{t}-1}^{n_{t}+1}, j=0,1, \ldots$, $\lambda_{t+2}-2$. To do this we look at the number of errors for $\varphi$ in the $j$-th column. If the number of errors is even (in particular if there are no errors) then we put

$$
\left.\psi\right|_{D_{j-n_{t}+n_{t-1}}^{n_{t}}}=b_{n_{t}-1}^{t}
$$

and $1+b_{n_{t-1}}^{t}$ otherwise. 
Of course this procedure leads to the definition of a Morse cocycle. Moreover $\psi$ admits an odd approximation with speed $0\left(1 / n^{1+t}\right)$, by (16) and (14).

It remains to prove that the cocycle $\varphi+\psi$ is not ergodic, i.e. that $T_{\varphi+\psi}$ is not ergodic. To this end we will define a sequence of sets $\left\{A_{t}\right\}, A_{t} \subset X \times \mathbf{Z}_{2}$ satisfying the assumptions of Lemma 1 . For every $t \geq 0$ we take $D^{n_{t}} \times 0$ and $D^{n_{t}} \times 1$.

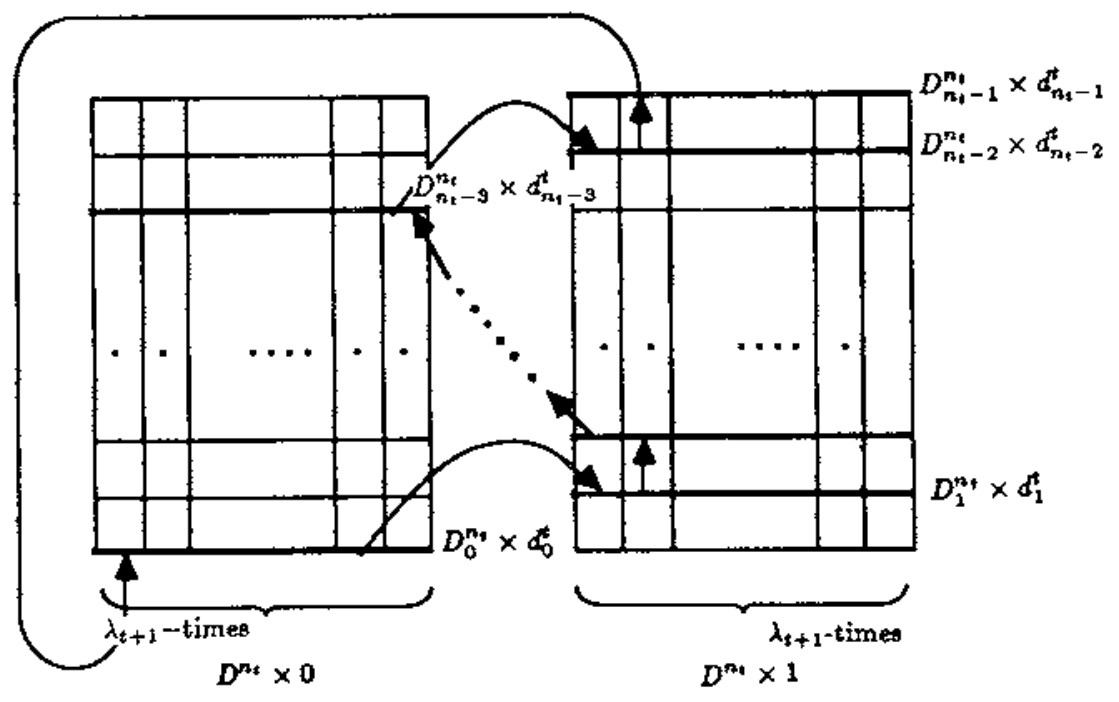

Picture 2

Put

$$
c_{i}^{t}=a_{i}^{t}+b_{i}^{t}, \quad i=0,1, \ldots, n_{t}-1, \quad t \geq 0 .
$$

Then by (16) and (12)

$$
\sum_{i=0}^{n_{i}-1} c_{i}^{t}=0
$$

We define the sets $A_{t}, t \geq 1$, putting

$$
A_{t}=\bigcup_{i=0}^{n_{t}-1}\left(D_{i}^{n_{2}} \times d_{i}^{t}\right),
$$

where $d_{0}^{t}=0$ and $d_{i}^{t}=c_{0}^{t}+\cdots+c_{i-1}^{t}, \quad i=1,2, \ldots, n_{t}$. It is clear that

$$
\tilde{\mu}\left(A_{\epsilon}\right)=\frac{1}{2} \text {. }
$$


Now we show that

$$
\sum_{t=1}^{\infty} \tilde{\mu}\left(A_{t+1} \triangle A_{t}\right)<\infty
$$

Remark that

$$
\tilde{\mu}\left(A_{t+1} \triangle A_{t}\right)=\frac{1}{n_{t}} .
$$

(The number of all levels $D_{k}^{n_{i+1}}, k=j \cdot n_{t}+i, \quad j=0, \ldots, \lambda_{t+1}-1, \quad i=$ $0, \ldots, n_{t}-1$ such that $\left.d_{k}^{t+1} \neq d_{i}^{t}\right)$. It follows from the definitions of $a_{i}^{t}$ and $b_{i}^{t}$ that $c_{k}^{t+1}=c_{i}^{t}$ whenever the $j$-th column contains no errors (with respect to $\varphi$ ) and each of the remaining columns contains an even number of the levels $D_{k}^{n_{t}+1}$ such that $c_{k}^{t+1} \neq c_{i}^{t}$. Because of (17) we conclude that $d_{k}^{t+1}=d_{i}^{t}$ whenever the $j$-th column contains no errors. The above considerations show that

$$
\tilde{\mu}\left(A_{t+1} \triangle A_{t}\right) \leq \frac{m_{t}}{\lambda_{t+1}}
$$

and then (13) and (15) imply

$$
\sum_{t=1}^{\infty} \tilde{\mu}\left(A_{t+1} \triangle A_{t}\right)<\infty .
$$

Now, we intend to estimate $\tilde{\mu}\left(T_{\varphi+\downarrow} A_{t} \triangle A_{t}\right)$. To this end, let us observe that:

$$
T_{\varphi+\psi}^{t}(x, i)=\left(T^{*} x,(\varphi+\psi)(x)+(\varphi+\psi)(T x)+\cdots+(\varphi+\psi)\left(T^{t-1} x\right) .\right.
$$

In other words, if $\varphi+\psi$ were constant on all levels of $D^{n s}$ (and equal to $c_{i}^{t}$ respectively) then we would get

$$
T_{\varphi+\psi}^{t}\left(D_{0}^{n_{t}} \times 0\right)=D_{t}^{n_{t}} \times\left(c_{0}^{t}+\cdots+c_{i-1}^{t}\right)=D_{1}^{n_{t}} \times d_{i}^{t}, t=1,2, \ldots, n_{t}-1
$$

and by (17)

$$
T_{\varphi+\psi}^{n_{t}}\left(D_{0}^{n_{t}} \times 0\right)=D_{0}^{n_{t}} \times 0
$$

(see picture 2). So, the above would mean $T_{\varphi+\psi}\left(A_{t}\right)=A_{t}$. However, $\varphi+\psi$ is not constant on the levels of $D^{n_{e}}$. By the argument we have just used it is easy to show that

$$
\tilde{\mu}\left(T_{\varphi+\psi} A_{t} \triangle A_{t}\right)=\frac{\varepsilon_{t}}{n_{t}^{e}}, \quad \varepsilon_{t} \longrightarrow 0 .
$$

Combining (18), (19) and (20) we see that the assumptions of Lemma 1 are satisfed and we conclude that $T_{\varphi+\psi}$ cannot be ergodic.

If we assume that $\varphi$ admits an even approximation with speed $0\left(\frac{1}{n^{1+\varepsilon}}\right)$ and that $\varphi$ is ergodic then we can repeat the foregoing proof with the only change that $b_{n:-1}^{t}$ is defined so that

$$
\sum_{i=0}^{n_{t}-1} b_{i}^{t}=0
$$

Therefore the proof of Theorem 1 is complete. 


\section{Factors of $Z_{2}$-extensions given by Morse cocycles}

We start with the definition of rigidity. Let $U:(Y, B, \nu) \circlearrowright$ be an ergodic transformation. $U$ is said to be rigid if there exists a sequence of positive integers $\left\{n_{i}\right\}, n_{i} \rightarrow \infty$ such that $U^{n_{i}} \rightarrow i d$ (the identity) in the weak topology, i.e. $\nu\left(U^{n}(A) \triangle A\right) \underset{i}{\rightarrow} 0$ for every $A \in B$.

Proof of Theorem 2: (In the proof we use notations from [14]). Let $x=b^{0} \times$ $b^{1} \times \ldots$ be a Morse sequence and let $(r, W, \nu)$ be a proper factor of $\left(r, O_{x}, \mu_{z}\right)$.

Let $\psi:\left(r, O_{z}, \mu_{x}\right) \longrightarrow(r, W, \nu), W \subset\{0,1\}^{z}$, establish a homomorphism. In order to prove that $r: W O$ is rigid it is enough to show that there is a generic point $w \in W$ such that for every $\varepsilon>0$ there is $s \in \mathbf{Z}$ such that

$$
\bar{d}\left(r^{\mathrm{s}} w, w\right)<\varepsilon,
$$

where $\tilde{d}\left(u, u^{\prime}\right)=\lim _{m} \inf \frac{1}{m} \operatorname{card}\left\{1 \leq i \leq m, u[i] \neq u^{\prime}[i]\right\}, u, u^{\prime} \in O_{x}$. Fix an $\varepsilon>0$. Then by the Birkhof Theorem there is a code $\varphi_{\varepsilon}: O_{x} \longrightarrow\{0,1\}^{2}$ (i.e. $\varphi_{\varepsilon} r=\tau \varphi_{\varepsilon}, \varphi_{\varepsilon}$ is measurable, $z[-k, k]=z^{\prime}[-k, k]$ implies $\left(\varphi_{c} z\right)[0\}=\left\{\varphi_{\varepsilon} z^{\prime}\right)[0]$, where $k=\left|\varphi_{c}\right|$ is the length of the code) such that

$$
\bar{d}\left(\psi z, \varphi_{x} z\right)<\frac{\varepsilon}{3} \text { for a.e. } z \in O_{x} .
$$

Then take $\delta, \quad 0<\delta<\varepsilon / 300\left\{2\left|\varphi_{\varepsilon}\right|+1\right)$ and fix $w \in W$. Since $\psi$ cannot be one-to-one, there are $z, z^{\prime} \in O_{x}, z \neq z^{\prime}$ such that

$$
\psi(z)=\psi\left(z^{\prime}\right)=w
$$

Then choosing a code $\varphi_{\delta}$ we can repeat the proof of Theorem 2 in [14] saying that there is an $s$ such that either

$$
\bar{d}\left(r^{s} z, z^{\prime}\right)<100 \delta
$$

or

$$
\bar{d}\left(r^{s} z, \overline{z^{4}}\right)<100 \delta
$$

All we have to prove that both (23) and (24) imply (21). First of all Remark the following property of codes.

Lemma 2. If $u, u^{t} \in O_{x}$ then

$$
d\left(\varphi_{\varepsilon} u, \varphi_{\varepsilon} u^{\prime}\right) \leq\left(2\left|\varphi_{\varepsilon}\right|+1\right) \cdot \bar{d}\left(u, u^{\prime}\right) .
$$

Proof: If $u[t-k, t+k]=u^{\prime}[t-k, t+k]$ then $\left(\varphi_{\varepsilon} u\right)[t]=\left(\varphi_{\varepsilon} u^{\prime}\right)[t]$. Hence if $\left(\varphi_{e} u\right)[t] \neq\left(\varphi_{e} u^{t}\right)[t]$ then it delivers at most $2\left|\varphi_{e}\right|+1$ places where $u$ and $u^{t}$ are different. 
Now

$$
\begin{aligned}
& \bar{d}\left(r^{s} w, w\right)=\bar{d}\left(r^{s} \psi(z), \psi\left(z^{\prime}\right)\right)=\bar{d}\left(\psi\left(r^{s} z\right), \psi\left(z^{\prime}\right)\right) \\
& \leq \bar{d}\left(\psi\left(r^{s} z\right), \psi_{\varepsilon}\left(r^{s} z\right)\right)+\bar{d}\left(\varphi_{\varepsilon}\left(r^{s} z\right), \varphi_{\varepsilon}\left(z^{\prime}\right)\right) \\
&+\bar{d}\left(\varphi_{\varepsilon}\left(z^{\prime}\right), \psi\left(z^{t}\right)\right) \stackrel{(22)}{\leq} \frac{2 \varepsilon}{3}+\bar{d}\left(\varphi_{\varepsilon}\left(r^{s} z\right), \varphi_{\varepsilon}\left(z^{t}\right)\right) \stackrel{(25)}{\leq} \frac{2 \varepsilon}{3} \\
&+\left(2\left|\varphi_{\varepsilon}\right|+1\right) \cdot \bar{d}\left(r^{s} z, z^{\prime}\right)<\varepsilon
\end{aligned}
$$

if (23) holds. If (24) holds then using (22) and (25) again we obtain

$$
\bar{d}\left(r^{s} w, \tilde{w}\right)<\varepsilon
$$

In both cases we can find a sequence $m_{3}$ such that either $r^{m} \rightarrow$ id or $r^{m} \cdot \rightarrow \sigma, \quad(\sigma(u)=\tilde{u})$. In the latter case $r^{2 m} \cdot \rightarrow i d$. This completes the proof of Theorem 2 .

Now applying the construction used in the proof of Theorem 1 we are able to indicate some factors of $\mathbf{Z}_{2}$-extensions determined by Morse cocycles. These factors will have a continuous part of the spectrum.

Assume that $T:(X, B, \mu) \circlearrowleft$ has r.p.p.s., $S_{p}(T)=G\left\{n_{t}, t \geq 0\right\}$ and let $\varphi: X \longrightarrow Z_{2}$ be a Morse cocycle given by

$$
\left.\varphi\right|_{D_{i}^{n_{t}}=a_{i}^{t}, \quad i=0,1, \ldots, n_{t}-2 .}
$$

We can define a sequence of blocks $\left\{\alpha^{t}\right\}, \quad\left|\alpha^{t}\right|=\lambda_{t}-1$ in the following way:

$$
\begin{gathered}
\alpha^{0}=\left(a^{0}[0], \ldots, a^{0}\left(\lambda_{0}-2\right]\right), \\
\alpha^{t+1}[j]=a^{i+1}\left[j \cdot n_{t}+n_{t}-1\right\}, \quad j=0,1, \ldots, \lambda_{t+1}-2 .
\end{gathered}
$$

The sequence of blocks $\left\{\alpha^{\ell}\right\}$ determines the Morse cocycle $\varphi$ completely. Now, let $s \geq 1$ be such that

$$
\left(s, n_{t}\right)=1, \quad t \geq 0
$$

Consider $\zeta_{s}:\left(Z_{s}, \nu_{s}\right) \bigcirc$ the cyclic rotation on $Z_{s}=\{0,1, \ldots, s-1\}$ with the uniform measure. In view of (26)

$$
T^{t}=T \times \varsigma_{s}: X \times \mathbf{Z}_{s} \longrightarrow X \times \mathbf{Z}_{s}
$$

is ergodic and has r.p.p.s., $S_{p}\left(T^{\prime}\right)=G\left\{s \cdot n_{t}, t \geq 0\right\}$. Define a function $\tilde{\varphi}: X \times$ $\mathbf{Z}_{\text {s }} \longrightarrow \mathbf{Z}_{2}$ by

$$
\tilde{\varphi}(x, i)=\varphi(x), \quad x \in X, \quad i \in \mathbf{Z}_{3} .
$$

Then we have a $Z_{2}$-extensions $\left(T^{\prime}\right)_{\ddot{\varphi}}=T_{\varphi} \times \zeta_{s}$ of $T^{\prime}$. Assume that $\varphi$ is oddly (or evenly) approximated with speed $0\left(\frac{1}{n_{q}^{1+e}}\right)$. Then $\tilde{\varphi}$ is approximated with the 
same speed. If follows from Theorem 1 that $\tilde{\varphi}$ can be modified by a coboundary cocycle i.e. by $\psi^{\prime}+\psi^{\prime} \circ T^{\prime}$ to get a Morse cocycle $\psi$. Then $T_{\varphi}$ is a factor of $T_{\psi}^{\psi}$. We intend to describe this passage from $T_{\varphi}$ to $T_{\psi}^{\prime}$ in a combinatorial language.

It is easy to see that the condition of the approximation of $\varphi$ with speed $0\left(\frac{1}{n_{1}^{\frac{1}{2}+e}}\right)$ implies

$$
\frac{1}{\lambda_{t+1}} \min \left(f r\left(0, \alpha^{t+1}\right), f r\left(1, \alpha^{t+1}\right)\right)=0\left(\frac{1}{n_{i}^{\varepsilon}}\right)
$$

where $f r\left(i, \alpha^{t+1}=\operatorname{card}\left\{j ; 0 \leq j \leq\left|\alpha^{t+1}\right| ; \alpha^{t+1}[j]=i\right\}\right.$. For every $t \geq 0$ we take the sets $D^{n_{t}} \times 0, D^{n_{t}} \times 1, \ldots, D^{n_{t}} \times(s-1)$. Then we can construct a $T^{\prime}$-tower of height $m_{t}=s \cdot n_{t}$

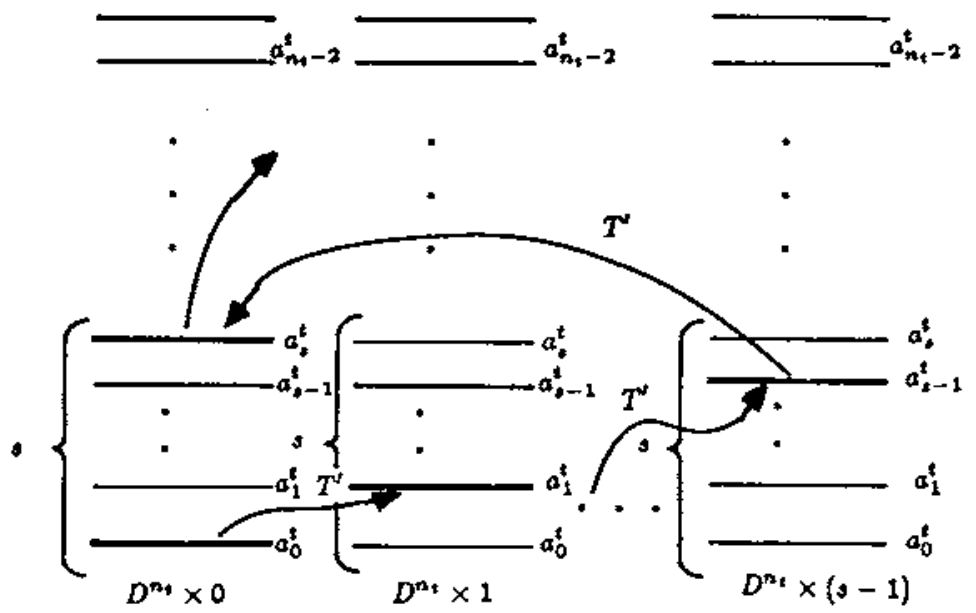

Picture 3.

We have

$$
\left(T \times s_{s}\right)\left(D_{j}^{n_{t}} \times i\right)=D_{j+1}^{n_{i}} \times(i+1),
$$

where the additions are taken $\bmod n_{t}$ and $\bmod s$ respectively. Let

$$
D_{k}^{m_{t}}=\left(T^{\prime}\right)^{k}\left(D_{0}^{n_{t}} \times 0\right), \quad k=0,1, \ldots, m_{t}-1 .
$$

Then (26) implies that each $D_{k}^{m_{2}}$ coincides with a level $D_{j}^{n_{2}} \times i$ for some $i, j$, $0 \leq j \leq n_{\mathrm{t}}-1,0 \leq i \leq s-1$, and this correspondence is one-to-one. Thus

$$
D_{0}^{m_{t}} \stackrel{T^{\prime}}{\longrightarrow} D_{1}^{m_{t}} \stackrel{T^{\prime}}{\longrightarrow} \ldots \stackrel{T^{\prime}}{\longrightarrow} D_{m_{t}-1}^{m_{t}} \stackrel{T^{\prime}}{\longrightarrow} D_{0}^{m_{t}},
$$


so $D^{m_{t}}$ is a $T^{\prime}$-tower of height $m_{t}$. If follows from the definition of $\tilde{\varphi}$ (see Picture 3) that

$$
\left.\tilde{\psi}\right|_{D_{k}^{m_{4}}}=\tilde{a}_{k}^{t}=a_{i}^{t}
$$

if $k=l \cdot n_{t}+i$ and $i=0,1, \ldots, n_{\mathrm{t}}-2, \quad l=0, \ldots, s-1$.

According to (27)

$$
\left.\tilde{\psi}\right|_{D_{k}^{m_{t}}}=\tilde{a}_{k}^{t}=a_{n_{\mathrm{t}}-1}^{t}
$$

if $k=l \cdot n_{t}+n_{t}-1, \quad l=0, \ldots, s-1$, where $a_{n t-1}^{t}=\tilde{\alpha}_{t+1}$ appears at $\alpha^{t+1}$ with frequency $>1-\frac{e_{t}}{n_{i}^{t}}, \varepsilon_{t} \rightarrow 0$. The symbols $\tilde{a}_{u}^{t+1}, 0 \leq u \leq m_{t+1}-1$ are the following. We write $u$ in the form

$$
u=l^{t} \cdot n_{t+1}+v \cdot n_{t}+r
$$

where $l^{t}=0,1, \ldots, s-1, v=0, \ldots, \lambda_{t+1}-1, r=0, \ldots, n_{t}-1$. Then

$$
\begin{cases}\tilde{a}_{u}^{t+1}=a_{i}^{t}, & \text { if } r<n_{t}-1, \\ \tilde{a}_{u}^{t+1}=\alpha^{t+1}[v], & \text { if } v<\lambda_{t+1}-1 \text { and } r=n_{t}-1 \\ \tilde{a}_{u}^{t+1}=a_{n_{t+1}-1}^{t+1}=\tilde{\alpha}^{t+2}, & \text { if } v=\lambda_{t+1}-1 \text { and } r=n_{t}-1 .\end{cases}
$$

We define $\psi$ on $D^{m_{0}}$ in an arbitrary way and suppose that $\psi$ have been defined on $D^{m_{i}}$ except for the level $D_{m_{i}-1}^{m_{1}}$. We should calculate the number of errors in each column of $D^{m_{t}}$. Comparing the symbols $\tilde{a}_{k}^{t}, 0 \leq k \leq m_{3}-1$ and $\tilde{a}_{t}^{t+1}, 0 \leq u \leq m_{t+1}-1$ (see Picture 4) from (28), (29) and (30) we can formulate the following algorithm of a construction of $\psi$ on $D_{m_{t}-1}^{m_{t}}$.

A) Define $\tilde{b}_{m_{t}-1}^{t}=\tilde{b}_{t}$ according to (16), i.e. if $\left.\psi\right|_{D_{k}^{m_{t}}}=\tilde{b}_{k}^{t}, 0 \leq k \leq m_{t}-1$ we chose $\tilde{b}_{i}$ in such a way that

$$
\sum_{k=0}^{m_{t}-1} \tilde{b}_{k}^{t}= \begin{cases}1, & \text { if the approximation is odd } \\ 0, & \text { if it is even. }\end{cases}
$$

B) Write the block $B^{t}$ of length $s \cdot \lambda_{t+1}$ as follows

$$
B^{t}=\underbrace{\alpha^{t+1}[0] \ldots \alpha^{t+1}[\lambda-2] \bar{\alpha}_{t+2}} \underbrace{\alpha^{t+1}[0] \ldots \alpha^{t+1}[\lambda-2] \tilde{\alpha}_{t+2}}, \quad \underbrace{\alpha^{t+1}[0] \ldots \alpha^{t+1}[\lambda-2] \bar{\alpha}_{t+2}} \cdots
$$

where $\lambda=\lambda_{t+1}$. 
C) Divide the block $B^{t}$ into $\lambda_{t+1}$ equal parts, i.e.

$$
B^{t}=B_{0} B_{1} \ldots B_{\lambda_{t+3}-1}, \quad\left|B_{j}\right|=s, \quad j=0,1, \ldots, \lambda_{t+1}-1
$$

and define $\psi$ on $D_{u}^{m_{t+1}}, u=t^{t} \cdot m_{t}+m_{t}-1, \quad l=0,1, \ldots, \lambda_{t+1}-2$ putting $\left.\psi\right|_{D_{t}^{m+1}}= \begin{cases}\tilde{b}_{t} & \text { if the number of the symbol }\left(1+\tilde{\alpha}_{t+1}\right) \text { in } B_{1} \text { is even } \\ \tilde{b}_{t}+1, & \text { otherwise }\end{cases}$

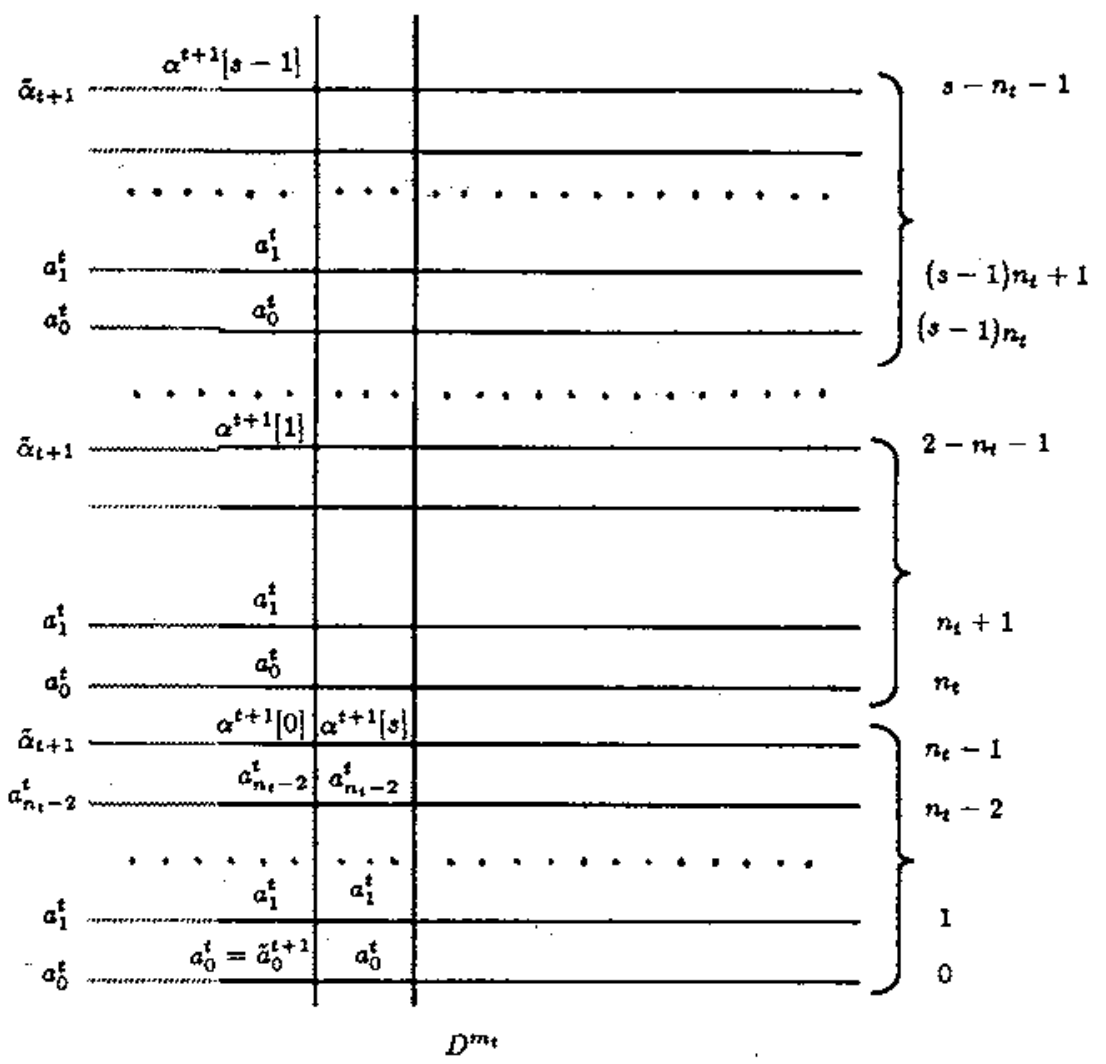

Picture 4

According to Theorem $1, T_{\varphi}^{\prime}$ is metrically isomorphic to $T_{\varphi} \times 5_{s}$.

\section{The rank power function}

Now, we intend to prove the existence of ergodic cocycles satisfying (5). In [11], Kwiatkowski and Rojet discovered a trichotomy concerning Morse shifts. Namely either

$$
C(T \varphi)=\left\{T_{\varphi}^{i} \circ \sigma^{j}\right\}, \quad i \in \mathbf{Z}, \quad j=0,1, \quad \sigma(x, i)=(x, i+1)
$$


or $T_{\varphi}$ is rigid. If the latter holds then either

$$
C\left(T_{\varphi}\right)=\text { weak closure of }\left\{\left(T_{\varphi}\right)^{n}, n \in \mathbf{Z}\right\},
$$

or

$$
C\left(T_{\varphi}\right) / \text { weak closure of }\left\{\left(T_{\varphi}\right)^{n}, n \in \mathbf{Z}\right\}=\{\mathrm{id}, \sigma\},
$$

where $C\left(T_{\varphi}\right)$ is the centralizer of $T$, i.e. the set of all automorphisms of $\left(X \times \mathbf{Z}_{2}, \tilde{B}, \tilde{\mu}\right)$ commuting with $T_{\varphi}$. No one of these possibilities is vacuous. Combining the Weak Closure Theorem [9] with the fact that the rank of Morse dynamical system is at most 2 one can easily prove that

$$
\operatorname{Ik}\left(T_{\varphi}\right)=2 \quad \text { iff } \quad \sigma \notin \text { weak closure }\left\{\left(T_{\varphi}\right)^{n}, n \in \mathbf{Z}\right\}
$$

as soon as $\varphi$ is a Morse cocycle.

Given $\varphi \in K$ we call the cocycle $\varphi+1$ (or $T_{\varphi+1}=T_{\varphi} \circ \sigma$ ) the completion of $\varphi$. The idea to prove (5) consists in the simple observation that a cocycle and its completion can have quite different properties. Take a Morse cocycle $\varphi$ such that $\varphi$ is evenly approximated with a given speed (at least $0\left(\frac{1}{n}\right)$ ) and moreover that

$$
\operatorname{rk}\left(T_{\varphi}\right)=2 .
$$

We recall that the paper $[11]$ assures an abundance of such cocycles. We assume here that $S_{p}(T)=G\left\{n_{t}, t \geq 0\right\}, n_{t}$ are odd. It is an easy observation that the completion of the $\varphi$ admits then an odd approximation with the same speed.

Therefore

$$
\operatorname{rk}\left(T_{\varphi+1}\right)=1
$$

and

$$
\mathrm{rk}\left(T_{\varphi+1}\right)^{2}=\mathrm{rk}\left(T_{\varphi}\right)^{2} \geq \operatorname{rk}\left(T_{\varphi}\right)=2 .
$$

Now, $\left(T_{\varphi}\right)^{2}=T_{\varphi+\varphi \circ}^{2}$ is ergodic and $\varphi+\varphi \circ T$ admits an even approximation with the same speed as $\varphi$. Because this speed is at least $0\left(\frac{1}{n}\right)$ the rank of $T_{\varphi}$ is at most 2 . Similar reasoning shows that

$$
\operatorname{rk}\left(T_{\varphi}\right)^{s}= \begin{cases}1, & \left(s, n_{t}\right)=1, s \text { is odd } \\ 2, & \left(s, n_{t}\right)=1, s \text { is even }\end{cases}
$$

The proof that m.s.m. $\left(T_{\varphi}\right)^{s}=1,\left(s, n_{t}\right)=1$ will follow from the following.

Lemma 3. Let $T$ be an ergodic automorphism with r.p.p.s., $S_{p}(T)=$ $G\left\{n_{t}, t \geq 0\right\}$. Assume that $\varphi$ admits an odd or even approximation with speed $O\left(\frac{1}{n}\right)$ and $\varphi$ is ergodic and nonconstant. Then $T_{\varphi}$ has simple spectrum.

Proof: The proof follows from the considerations from [7]. 
Remark. If $\mathrm{rk}\left(T_{\varphi}\right)=2$ and $\varphi$ admits an even approximation with speed $o\left(\frac{1}{n^{1+\theta}}\right)$ then, of course, $T_{\varphi}$ and $T_{\varphi+1}$ cannot be isomorphic $\left(\operatorname{rk}\left(T_{\varphi+1}\right)=1\right)$. Actually, they cannot be spectrally isomorphic. Indeed, $\left(T_{\varphi+1}\right)^{m_{t}} \longrightarrow \sigma$ for a sequence $\left\{m_{t}\right\}[9]$. If an infinity of the $m_{t}$ 's were even then $\left(T_{\varphi}\right)^{m_{*}}=$ $\left(T_{\varphi+1}\right)^{m_{t}} \rightarrow \sigma$ and from (33) $T_{\varphi}$ would have rank 1 . To avoid the contradiction we are forced to assume that the $m_{t}$ 's are odd. But then

$$
\left(T_{\varphi}\right)^{m_{t}} \rightarrow \text { id and }\left(T_{\varphi+1}\right)^{m_{t}} \nrightarrow \mathrm{id} .
$$

So $T_{\varphi}$ and $T_{\varphi+1}$ cannot be spectrally isomorphic.

Remark. It is interesting to know whether the oscilation of the rank power function is typical for $\mathbf{Z}$-cocycles. But it is not the case, as the following shows. First of all we notice that

$$
\operatorname{rk}\left(T_{\varphi}\right)^{2}=2 \quad \text { iff } \quad \operatorname{rk}\left(T_{\varphi+1}\right)=2
$$

as soon as $\varphi$ admits an odd approximation with speed $o\left(\frac{1}{n^{1+*}}\right)$. Indeed, if $\operatorname{rk}\left(T_{\varphi}\right)=1=\mathrm{rk}\left(T_{\varphi+1}\right)$, then

$$
\exists m_{t} \quad\left(T_{\varphi}\right)^{m_{t}} \longrightarrow \sigma
$$

and

$$
\exists m_{t}^{\prime} \quad\left(T_{\varphi+1}\right)^{m_{t}^{\prime}} \longrightarrow \sigma .
$$

Since (33) holds, it is enough to prove that $m_{t}$ or $m_{t}^{t}$ are even for an infinity of t's. If this is not the case, then $m_{t}, m_{t}^{t}$ are odd. We can also assume $m_{t}-m_{t}^{\prime} \nearrow \infty$ by passing to subsequences. Then

$$
\left(T_{\varphi}\right)^{m_{t}} \circ\left(T_{\varphi+1}\right)^{-m_{i}^{\prime}} \longrightarrow \sigma \circ \sigma^{-1}
$$

so

$$
\left(T_{\varphi}\right)^{m_{1}} \circ\left(T_{\varphi}\right)^{-m^{\prime} \circ \sigma \longrightarrow \sigma \circ \sigma^{-1}}
$$

which implies

$$
\left(T_{\varphi}\right)^{m_{t}-m_{i}} \longrightarrow \sigma
$$

and

$$
\left(T_{\varphi}\right)^{2 s_{t}} \longrightarrow \sigma, \text { where } 2 s_{t}=m_{t}-m_{t}^{\prime} .
$$

If $\operatorname{rk}\left(T_{\varphi+1}\right)=2$, then

$$
2 \geq \operatorname{sk}\left(\left(T_{\varphi}\right)^{2}\right)=\operatorname{rk}\left(\left(T_{\varphi+1}\right)^{2}\right) \geq \operatorname{rk}\left(T_{\varphi+1}\right)=2 .
$$

Now the set

$$
A=\left\{\varphi \in K, \operatorname{rk}\left(T_{\varphi}\right)=1\right\}
$$


is residual because it contains all cocycles oddiy approximated with speed o $\left(\frac{1}{n}\right)$. Therefore the set $A+1=\{\varphi+1, \varphi \in A\}$ is residual. Whence

$$
A \cap(A+1)=\left\{\varphi \in \mathcal{K}, \mathrm{rk}\left(T_{\varphi}\right)=1=\mathrm{rk}\left(T_{\varphi+1}\right)\right\}
$$

is residual. This fact and (34) give that the rank power function being constant (and equal 1 ) is a typical property for $K$.

Example. The next problem we intend to deal with here is the problem of lifting roots. It is well-know how to calculate roots for $T$ with r.p.p.s., $S_{p}(T)=G\left\{n_{t} ; t \geq 0\right\}$. It $\left\{s, n_{t}\right)=1, t \geq 0$ then $T^{s}$ and $T$ are isomorphic because they have r.p.p.s. and they have the common sequence $\left(D^{n_{i}}\right) / \mathrm{B}$. It would be interesting to know whether a sufficiently high speed of approximation of $\varphi$ assures the existence of some roots. However the results of [11] show that this supposition cannot be true.

We take the Morse sequence

$$
x=b^{0} \times b^{1} \times \ldots
$$

where

$$
b^{t}=\overbrace{0101 \ldots 01}^{\mu_{t}} \overbrace{1010 \ldots 101}^{\mu_{t+1}}, t \geq 0 .
$$

Assume that

$$
\sum_{0}^{\infty} \frac{1}{\mu_{t}}<\infty
$$

Put

$$
\lambda_{t}=2 \mu_{t}+1, \quad n_{t}=\lambda_{0} \cdot \ldots \cdot \lambda_{t}, \quad c_{t}=b^{0} \times \cdots \times b^{t}, t \geq 0
$$

and define blocks $\hat{c}_{t},\left|\hat{c_{t}}\right|=n_{t}-1$ putting

$$
\hat{c_{t}}[i]=c_{t}[i+1]+c_{t}[i] \quad \text { in } \mathbf{Z}_{2}, i=0,1, \ldots, \lambda_{t}-2 .
$$

Let $\Delta$ be the group of all $n_{t}$-adic numbers i.e.

$$
\triangle=\left\{g ; g=\sum_{0}^{\infty} g_{t} \cdot n_{t-1}, 0 \leq g_{t} \leq \lambda_{t}-1, n_{-1}=1\right\},
$$

and let $\mu$ be the Haar measure of $\triangle$ and $T$ be the rotation on $\hat{1}=(1,0,0, \ldots)$. For every $t \geq 0$ we have a $T$-power $D^{n}$, in $\Delta$ such that

$$
D_{i}^{n_{t}}=\left\{g ; \sum_{u=0}^{t} g_{u} \cdot n_{t s-1}=1\right\}, \quad i=0,1, \ldots, n_{t}-1 .
$$

The sequence $x$ determines a Morse cocycle $\varphi$ on $\triangle$ by

$$
\left.\varphi\right|_{D_{i}^{n_{t}}=\hat{\epsilon}_{t}[i], \quad i=0,1, \ldots, n_{t}-2 .}
$$


In $[11]$ the centralizer of $T_{\varphi}$ has been described. Each $S \in C\left(T_{\varphi}\right)$ has a form

$$
S(g, i)=\left(g+g_{0}, i+f(g)\right)
$$

where $g_{0} \in \triangle$ and $f: \triangle \longrightarrow \mathbf{Z}_{2}$ satisfy the condition

$$
\varphi\left(g+g_{0}\right)+f(g)=f(g+\hat{1})+\varphi(g) .
$$

We will show that for every $k>1$ there exists no $S \in C\left(T_{\varphi}\right)$ such that $S^{k}=T_{\varphi}$. Remark that

$$
S^{k}(g, i)=\left(g+k \cdot g_{0}, i+f(g)+\cdots+f\left(g+(k-1) g_{0}\right)\right) .
$$

Then $S^{k}=T_{\varphi}$ iff $k \cdot g_{0}=\hat{1}$ and

$$
f(g)+\cdots+f\left(g+(k-1) g_{0}\right)=\varphi(g)
$$

where $g_{0}$ and $f$ satisfy (35).

Notice that there exists $g_{0} \in \triangle$ with $k \cdot g_{0}=\hat{1}$ iff $\left(k, n_{t}\right)=1$. We will denote such $g_{0}$ by $\hat{1} / k$. Now we prove that for $k>2,\left(k, n_{t}\right)=1, \hat{1} / k$ does not satisfy (35) and $\hat{1} / 2$ satisfies (35) but the corresponding function $f$ does not satisfy (36).

Now, let $k>2,\left(k, n_{t}\right)=1, t=0,1, \ldots$ If $\hat{1} / k=\left\langle l_{t}\right)_{0}^{\infty}, 0 \leq l_{t} \leq n_{t}-$ $1, l_{t+1} \equiv l_{t}\left(\bmod n_{t}\right)$ then $l_{t}$ is one of the numbers

$$
\frac{n_{t}+1}{k}, \frac{2 n_{t}+1}{k}, \ldots, \frac{(k-1) n_{t}+1}{k} .
$$

For such a number $l_{t}$ we have

$$
\frac{l_{t}}{n_{t}} \geq \frac{1}{k}, 1-\frac{l_{t}}{n_{t}} \geq \frac{1}{2 k} \text { and }\left|\frac{l_{t}}{n_{t}}-\frac{1}{2}\right| \geq \frac{1}{3 k}
$$

for $t$ large enough.

If $\hat{1} / k$ is represented as a series $\sum_{0}^{\infty} \overline{g_{t}} \cdot n_{t-1}$, then the inequalities

$$
\left|\frac{l_{t}}{n_{t}}-\frac{\overline{g_{t}}}{\lambda_{t}}\right| \leq \frac{1}{\lambda_{t}}-\frac{1}{n_{t}} \leq \frac{1}{2 \lambda_{t}}
$$

imply

$$
\frac{\overline{g_{t}}}{\lambda_{t}} \geq \frac{1}{4 k}, \quad 1-\frac{\overline{g_{t}}}{\lambda_{t}} \geq \frac{1}{4 k}, \quad\left|\frac{g_{t}}{\lambda_{t}}-\frac{1}{2}\right| \geq \frac{1}{4 k}
$$

for $t$ large enough. On the other hand it is easy to remark that the inequalities

$$
\frac{g_{i}}{\lambda_{i}}>\delta, 1-\frac{g_{i}}{\lambda_{i}}>\delta \text { and }\left|\frac{g_{i}}{\lambda_{i}}-\frac{1}{2}\right|>\delta, 0 \leq g_{i} \leq \lambda_{i}-1
$$


imply

$$
d\left(b^{t} b^{t}\left[g_{t}, g_{t}+\lambda_{t}-1\right] b^{t}\right) \geq \delta
$$

Moreover, (38) is valid if we replace $b^{t} b^{t}$ by $b^{t} \overline{b^{t}}, \overline{b^{t}} b^{t}$ and $\overline{b^{t}} \overline{b^{t}}$. In view of Theorem 1 in $[11],(37)$ and (38) $g_{0}=\hat{1} / k$ does not satisfy (35).

Now take $\hat{1} / k$. Then $\frac{\hat{i}}{k}=\left(\frac{n_{t}+1}{2}\right)_{0}^{\infty}=\left(\mu_{0}+1\right)+\sum_{t=1}^{\infty} \mu_{t} \cdot n_{t-1}$. It is clear that

$$
d\left(b^{t} \tilde{b}^{t}\left[\mu_{t}+1, \mu_{t}+\lambda_{t}\right], b^{t}\right)=\sum_{0}^{\infty} \frac{1}{\lambda_{t}}<\infty .
$$

Applying again $[\mathbf{1 1}]$ we obtain that (35) is satisfied with $g_{0}=\hat{1} / 2$ and the function $f=\lim f_{t}$, where

$$
f_{t}(g)=c_{t} \tilde{c}_{t}\left[\frac{n_{t}+1}{2}+j_{t}\right]+c_{t}\left[j_{t}\right], g=\left(j_{t}\right), 0 \leq j_{t} \leq n_{t}-1
$$

Next we have

$$
\begin{aligned}
f_{t}(g)+f_{t}(g+\hat{1} / 2) & =\hat{c}_{t}\left[j_{t}+1\right]+c_{t}\left[\frac{n_{t}+1}{2}+j_{t}\right]+c_{t}\left[\frac{n_{t}+1}{2}+j_{t}\right]+c_{t}\left[j_{t}\right]= \\
& =\overbrace{c_{t}\left[j_{t}+1\right]+c_{t}\left[j_{t}\right]+1}^{\varphi(g)}, \quad \text { for } g=\left(j_{t}\right) \text { and } j_{t} \leq \frac{n_{t}-3}{2} .
\end{aligned}
$$

We conclude that (36) does not hold and $T_{\phi}$ has no roots of any degree. At the same time the function $\varphi$ admits an odd approximation with the speed $o\left(\frac{1}{\lambda_{t+1}} \cdot \frac{1}{n_{t}}\right)$.

Taking suitable $\lambda_{t}$ 's we can get Morse cocycles $\varphi$ 's admitting an approximation with an arbitrarily high speed.

\section{References}

1. G. Christol, T. KamaE, M. Mendes-France, G. Rauzy, Suites algébraiques, automates et substitutions, Bull. Soc. Math. France 108 (1980), 401-420 (in French).

2. E. COVEN, M. KEANE, The structure of substitution minimal sets, Trans. Amer. Math. Soc. 62 (1971), 89-102.

3. N. FRIEDMAN, P. Gabriel, J. KING, An invariant on rigid rank-1 transformations, preprint.

4. S. GLASNER, D. RUDOLPH, Uncountable many topological models for ergodic transformations, Erg. Th. Dyn. Syst. 4 (1984), 233-236. 
5. H. HELSON, Analyticity on compact abelian groups, Algebra in Analysis, ed. J. H. Williamson, Academic Press (1975), 1-62.

6. H. HeLsON, W. PARRY, Cocycles and spectra, Arkiv för Mat. 16 (1978), 195-206.

7. A.B. KATCK, A.M. STEPIN, Approximation in ergodic theory, Uspekhi Mat. Nauk 22 no. 5 (1967), 81-106 (in Russian).

8. R. KeAne, Generalized Morse sequences, Z. Wahr. Verw. Geb. 10 (1968), 335-353.

9. J. KING, The commutant is the weak closure of the powers, for rank-1 transformations, Erg. Th. Dyn. Syst. 6 (1986), 363-385.

10. J. KWIATKOWSKI, Isomorphism of regular Morse dynamical systems, Studia Math. 62 (1982), 59-89.

11. J. KWLATKOWSKI, T. ROJEK, the centralizer of Morse shifts induced by arbitrary blocks, Studia Math. (to appear).

12. M. LEMAṄCZYK, The centralizer of Morse shifts, Ann. Univ. ClermontFerrand 87 (1985), 43-56.

13. M. LeMÁ்̃zYK, Toeplitz $\mathbf{Z}_{2}$-extensions, Ann. H. Poincaré Inst. (to appear).

14. M. LEMAŃCZYK, Ergodic $\mathbf{Z}_{2}$-extensions over rational pure point spectrum, category and homomorphisms, Comp. Math. (to appear).

15. M. LEMÁ்czYK, M. K. MEntzen, Generalized Morse sequences on $n$-symbols and $m$-symbols are not isomorphic, Bull. Pol. Ac. Sc. 33 no. 5-6 (1985), 239-245.

16. J. MATHEW, M. G. NADKORNI, A measure-preserving transformation whose spectrum has Lebesgue component of multyplicity two, Bull. Lon. Math. Soc. 16 (1984), 402-406.

17. D. NEWTON, On canonical factors of ergodic dynamical systems, J. Lon. Math. Soc. 19 (1978), 129-136.

18. W. PARRY, Compact abelian group extensions of discrete dynamical systems, Z. Wahr. Verw. Geb. 13 (1969), 95-113.

19. W. PARRY, Topics in Ergodic Theory, C.U.P. (1981).

I. Filipowicz: Institute of Mathematics

Pedagogical College Chodkiewicza 30,

85-064 Bydgoszcz, POLAND.

J. Kwiatkowski: Institute of Mathematics

Nicholas Copernicus University

uI. Chopina 12/18, 87.100 Torun, POLAND.

M. Lemariczyk: Institute of Mathematics

Nicholas Copernicus University

ul. Chopina 12/18, 87-100 Torun, POLAND.

Rebut el 26 de Maig de 1987 